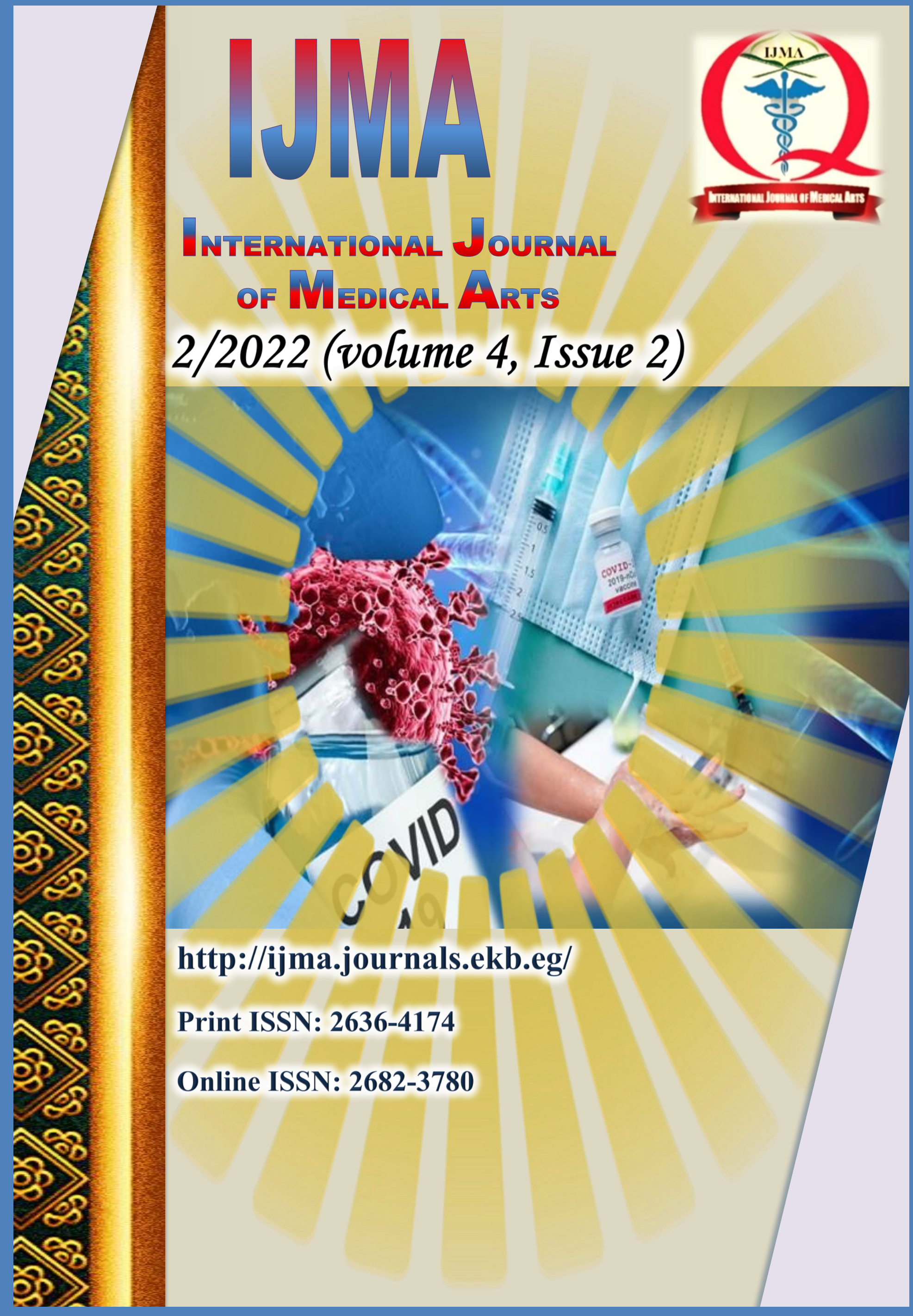





Available online at Journal Website
https://ijma.journals.ekb.eg/
Main Subject [Gynecology and Obstetrics]

Original Article

\title{
Bilateral Ovarian Huge Benign Papillary Serous Tumor in a Teenage Girl: A Case Report
}

\author{
Anand Dineshkumar Bhatt ${ }^{[1]}$; Vishal Chavda ${ }^{[2]}$
}

${ }^{1}$ Department of Obstetrics and Gynecology, Sardar Women's Hospital, Ahmadabad, Gujarat, India.
${ }^{2}$ Division of Anaesthesia, Sardar Women's Hospital, Ahmedabad, Gujarat, India.

\begin{tabular}{|l||}
\hline Article information \\
Received: \\
Accepted: \\
DOI: $13-11-2021$ \\
22-01-2022 \\
\hline \hline *Corresponding author \\
Email: chavdavishal2@ @mail.com \\
\hline \hline Citation: Bhatt AD, Chavda V. Bilateral \\
Ovarian Huge Benign Papillary Serous \\
Tumor in a Teenage Girl: A Case Report. \\
IJMA 2022; 4 [2]: 2133-2135. doi: \\
10.21608/ijma.2022.105923.1392 \\
\\
\hline
\end{tabular}

ABSTRACT

Objective: Ovarian cysts are a very common gynaecological problem in adolescents and teenagers. The majority of ovarian cysts are benign, with only a small percentage of cases progressing to malignancy. Ovarian serous cystadenoma is uncommon in children, and it can progress to benign or malignant papillary serous tumours with oncological changes.

Case report: The 18-year-old female patient arrived at the clinic complaining of abdominal heaviness and pain. Some cystic non-mobile mass was palpable up to $6 \mathrm{~cm}$ above the umbilical level on abdominal examination. On ultrasonographic examination, a cystic mass measuring $17 \times 10 \times 8 \mathrm{~cm}$ was discovered in the right ovary and was not seen separately; 7 x $6 \mathrm{~cm}$ cystic swelling was discovered in the left ovary. Serum CEA and CA 125 levels were measured and found to be 1.29 and 14.9, respectively, for further oncoanalysis, both of which were within normal limits. The tube was gently separated from the mass using a skilled surgical technique, and the cystic mass from both ovaries was removed. The cystic masses were sent for histopathological examination, which revealed that they were benign papillary serous tumours.

Conclusion: A benign papillary serous tumour in a teenage girl is a rare and difficult finding. Cysts are common, but papillary serous tumours are unusual. Careful USG and early diagnosis of such cysts, as well as skilful surgical removal of such cysts, can improve the quality of life in teenagers.

Keywords: Benign Papillary Serous Tumor; Ovarian Serous Cystadenoma; Ultrasonographic [USG] Examination; Teenage Girl; Ovarian Cancer.

This is an open-access article registered under the Creative Commons, ShareAlike 4.0 International license [CC BY-SA 4.0] [https://creativecommons.org/licenses/by-sa/4.0/legalcode. 


\section{INTRODUCTION}

A serous tumour is an ovarian neoplasm that belongs to the surface epithelial-stromal tumour group and is primarily derived from Mullerin epithelium. They are mostly common neoplasms with a high proclivity for bilateralism. They are responsible for half of all ovarian tumors. Approximately $60 \%$ of them are benign [cystadenoma] in nature, $10 \%$ are borderline, and $30 \%$ are malignant [cystadenocarcinoma] in nature. Ovarian cysts can occur at any age, but they are most common during pubescence, a hormonally active period in girls' development ${ }^{[1]}$.

The two most common types of functional cysts formed during ovulation are follicular and corpus luteum cysts. Ovarian cysts are typically non-neoplastic and grow infrequently. The diagnostic ultrasonography imaging technique allows for the early detection of ovarian cysts and aids in the provision of appropriate medical care. Ovarian cysts occasionally expand in three dimensions without causing any symptoms. A few cases of giant ovarian cysts have been reported sporadically in the literature ${ }^{[2]}$

Huge papillary projections with serous cyst adenoma were observed in a case study of the gross and microscopic characterization of a benign ovarian cyst in an 18-year-old girl.

\section{Case Presentation}

An 18-year-old female patient was brought to the clinic complaining of abdominal heaviness and pain. A cystic nonmobile mass was palpable up to $6 \mathrm{~cm}$ above the umbilical level during an abdominal examination. Vitals were within the normal range. A cystic mass measuring $17 \times 10 \times 8 \mathrm{~cm}$ was discovered during an ultrasonographic examination. The right ovary was not visible separately. Another 7 × $6 \mathrm{~cm}$ cystic swelling arising from the left ovary was discovered. Serum CEA and CA 125 levels were measured and found to be 1.29 and 14.9, respectively, which are within normal limits. The patient was taken to the operating room after completing preoperative fitness.

The Pfannenstiel incision was performed with the patient's age and cosmetic preferences. Exteriorization of cystic mass was possible with good anesthetic support and muscle relaxation. The mass was arising from the rightside ovarian tissue, and the right fallopian tube was stretched out like a very thin strip over the mass. The tube was gently separated from the mass using skilled surgical technique, and a cystic mass measuring approximately 19 x 15 x $10 \mathrm{~cm}$ was removed [Figure-1].

The ovary and tubes on the right side were saved. The right fallopian tube was stretched to a length of about 18$20 \mathrm{~cm}$. The serosa of tube was sutured with Vicryl 4-0 so that the tubal opening was bear right ovary and towards the ovary. The ovarian tissue was salpingopexied with skill and sterility. On the left side, an 8 x $7 \mathrm{~cm}$ cystic mass was separated and the tube and ovary were preserved; the wound was closed, and the post-operative period was uneventful.

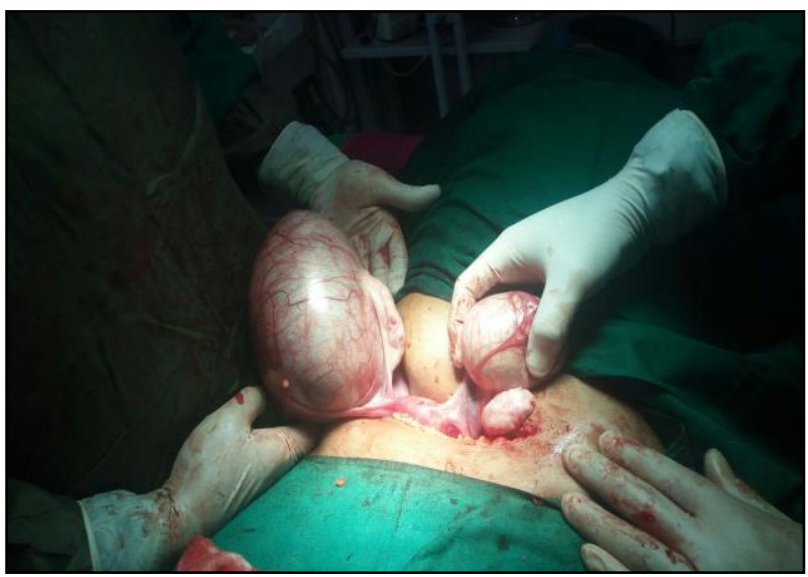

Figure [1]: Bilateral ovarian serous papillary tumor

Both cystic masses were sent for histopathological examination, which revealed that they were benign papillary serous tumors on both sides. After one week, the stitches were removed and the postoperative follow-up was completed. The patient returned to his normal routine.

\section{DISCUSSION}

The most common symptoms of ovarian cysts are pelvic pain, nausea, vomiting, and a history of previous episodes of similar pain and a low-grade fever ${ }^{[3,4]}$.

Time is of the essence when it comes to the differential diagnosis of functional cysts in pubertal girls. A functional cyst is characterized by being unilocular, anechoic, and with thin borders. The second differential diagnosis for ovarian cysts is omental cysts. Omental cysts can affect people of all ages, but they are more common in children and adolescents. Pancreatic pseudo cysts, mesenteric cysts, urinary retention, bladder diverticulum, hydronephrosis, cystic lymphangiomas, choledochalcysts, splenic cysts, multicystic dysplastic kidney, gastrointestinal duplication cysts, and broad uterine tumour are all possible diagnoses ${ }^{[5]}$.

While the cause of malignant transformation is unknown, clonal origin of benign cancer antigen [CA], borderline tumour, and low-grade epithelial carcinomas is suspected ${ }^{[3]}$.

The patient's age, the size and shape of the cyst, and the patient's menopausal status all play a role in ovarian cyst management. The surgical options for cyst removal are laparotomic or laparoscopic cyst excision or cystectomies with oophorectomy. In addition, the contralateral ovary should be examined, and if it appears abnormal, a frozen segment can be used to determine whether it should be removed or not ${ }^{[6]}$.

According to some authors, the best method is intact cyst removal with incremental rolling of the mass off the inferior vena cava. Progressive decompression, on the other hand, prevents cyst capsule rupturing and increases 
dissemination, according to Hunter et al. ${ }^{[7]}$. A possible complication of repeated paracentesis is intra-peritoneal spillage of cyst components. If the cyst is malignant, tumour seeding of the peritoneal cavity or paracentesis tract may occur. Infection, bleeding, and increased peritoneal adhesions are all risks of repeated paracentesis, making cyst removal more difficult ${ }^{[8]}$.

In an emergency, when waiting for tumour marker results is not an option, the choice between laparoscopy and laparotomy can be made based on the size and shape of the swollen ovary ${ }^{[6-8]}$. Low malignant recurrences in the adult patient population have been identified for more than ten years after initial surgery, so it's probably safe to say that diagnosed adolescence should be followed until they reach adulthood. Long-term follow-up can be difficult, as evidenced by patients who have gone missing for years, but this does not influence the decision to perform a conservative surgical procedure to preserve a patient's fertility ${ }^{[9,10]}$.

A gynecologist should perform a histopathological examination and blood biochemistry if there is a family history of ovarian cancer. Germ cell tumors are the most common cause of giant ovarian masses in children, but epithelial tumors should not be overlooked in the differential diagnosis.

\section{Key Clinical Message:}

Ovarian cysts are frequently misdiagnosed as benign tumors or are incorrectly suspected to be malignant. Careful ultrasound examination, observing echogenicity, vascularity, the presence of solid components, pockets, and mobility, aids in proper diagnosis and must be performed. In addition, tumour markers such as CA125, CEA, and others aid in either strengthening or eliminating the diagnosis. An MRI or CT scan could be extremely useful in making a diagnosis. During the surgical procedure, the cystic masses are so large that the fallopian tubes are stretched to the point of containing only thin strip-like structures. It is sometimes difficult for the surgeon to only perform a cystectomy and save the ovary and tubes because it is extremely difficult to separate the two structures and salpingoopherectomy is unavoidable, but the ovary and tubes can be saved as well with patience and careful surgical technique. Every effort must be made to save the fertility-providing organs, especially in the case of adolescent or unmarried girls. In the case of cancer, a frozen section can be sent for confirmation of the diagnosis and further surgical intervention.

Recurrent Follow-Up Protocol: Careful follow-up with proper ultrasound must be done every month for three months, then every three months for a year, and once a year for the next five years.

\section{Acknowledgement: None}

\section{Disclosure for informed Consent:}

Proper informed and written consent in local understandable language was taken from the patient regarding publication of the same case.

Author Contribution: Vishal Chavda [VC] Prepared Case report, analysed case report, finalised manuscript draft, assisted in anaesthesia, Anand Bhatt [AB] analysed final report, Performed Gynaecological surgery

Funding: None to Declare

\section{REFERENCES}

1. Alpendre F, Pedrosa I, Silva R, Batista S, Tapadinhas P. Giant Paratubal cyst presenting as adnexal torsion: A case report. Case Rep Womens Health. 2020 May 19;27: e00222. doi: 10.1016/j.crwh.2020.e00222.

2. Zvizdic Z, Bukvic M, Murtezic S, Skenderi F, Vranic S. Giant Paratubal Serous Cystadenoma in an Adolescent Female: Case Report and Literature Review. J Pediatr Adolesc Gynecol. 2020;33[4]:438-440. doi: 10.1016/ j.jpag.2020.03.010.

3. Carbonnel M, Layoun L, Poulain M, Tourne M, Murtada R, Grynberg M, Feki A, Ayoubi JM. Serous Borderline Ovarian Tumor Diagnosis, Management and Fertility Preservation in Young Women. J Clin Med. 2021 Sep 18;10 [18]:4233. doi: 10.3390/jcm10184233.

4. Limaiem F, Lekkala MR, Mlika M. Ovarian Cystadenoma. 2021 Jul 20. In: StatPearls [Internet]. Treasure Island [FL]: StatPearls Publishing; 2021 Jan-. PMID: 30725635

5. Ben-Mussa A, McCluggage WG. Ovarian seromucinous cystadenomas and adenofibromas: first report of a case series. Histopathology. 2021 Feb;78[3]:445-452. doi: 10.1111/his.14240.

6. Samartzis K, Kathopoulis N, Loutradis D, Protopapas A. Do techniques of surgical management of ovarian endometrioma affect ovarian reserve? A narrative review. J Obstet Gynaecol. 2021:1-7. doi: 10.1080/ 01443615. 2021.1962817.

7. Hunter DJS. Management of a massive ovarian cyst. Obstet Gynecol 1980; 56: 254-255

8. Biçer S, Erkul Z, Demiryilmaz I, Peker N. A 9-kg ovarian mucinous cystadenoma in a 14-year-old premenarchal girl. Am J Case Rep. 2014 Aug 1; 15:326-9. doi: 10.12659/AJCR.890862.

9. Karaman A, Azili MN, Boduroğlu EC, Karaman I, Erdoğan D, Cavuşoğlu YH, Aslan MK, Cakmak O. A huge ovarian mucinous cystadenoma in a 14-year-old premenarchal girl: review on ovarian mucinous tumor in premenarchal girls. J Pediatr Adolesc Gynecol. 2008 Feb;21[1]:41-4. doi: 10.1016/j.jpag.2007.09.005.

10. Vicente AP, Gomes AS, Jokubkiene L, Sladkevicius P. Ovarian borderline tumor presenting as ovarian torsion in a 17-year-old patient: a case report. J Med Case Rep. 2021 Jan 12;15[1]:7. doi: 10.1186/s13256-020-02597-1. 


\section{2) 12022}

international Journal https://ijma.journals.ekb.egl Print ISSN: 2636-4174 Online ISSN: 2682-3780

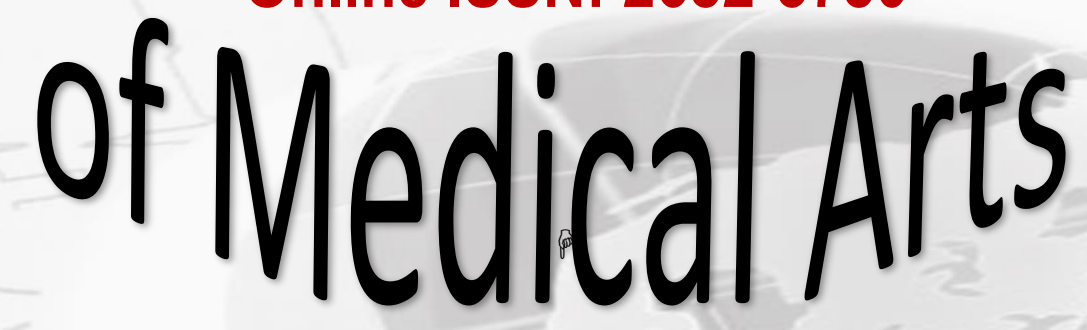

